\title{
ARTICLE
}

\section{A method for the prediction of the dose rate distribution in a primary containment vessel of the Fukushima Daiichi Nuclear Power Station}

\author{
Keisuke Okumura $^{a}$, Eka Sapta Riyana ${ }^{\mathrm{a}}$, Wakaei Sato ${ }^{\mathrm{a}}$, Hirobumi Maeda ${ }^{\mathrm{a}}$, Jun-ichi Katakura ${ }^{\mathrm{b}}$, So Kamada ${ }^{\mathrm{c}}$, \\ Malcolm J. Joyce ${ }^{\mathrm{d}}$ and Barry Lennox ${ }^{\mathrm{e}}$ \\ ${ }^{a}$ Japan Atomic Energy Agency, 790-1 Motooka, Ohtsuka, Tomioka-machi, Futaba-gun, Fukushima-ken, 979-1151, Japan; ${ }^{\circ}$ Nagaoka \\ University of Technology, 1603-1 Kamitomioka-machi, Nagaoka-shi, Niigata-ken, 940-2188, Japan; ${ }^{c}$ National Maritime Research \\ Institute, 6-38-1 Shinkawa, Mitaka-shi, Tokyo, 181-0004, Japan; ${ }^{d}$ Lancaster University, Bailrigg, Lancaster, United Kingdom, \\ ${ }^{e}$ The University of Manchester, Oxford Rd, Manchester, United Kingdom
}

We propose a method to predict the dose rate distribution in a primary containment vessel of Unit 1 of the Fukushima Daiichi Nuclear Power Station (1F) using numerical calculations and measured data.

Keywords: Fukushima Daiichi; dose rate; primary containment vessel; fuel debris; decommissioning; severe accident; fuel burnup; activation; photon transport; Monte Carlo; PHITS; PCV investigation

\section{Introduction}

In order to accelerate the retrieval of fuel debris at the Fukushima Daiichi Nuclear Power Station (1F), it is important to know the dose rate distribution in the primary containment vessel (PCV). To grasp the current situation inside a PCV, severe accident (SA) analyses and PCV investigations using robots have been carried out by the International Research Institute for Nuclear Decommissioning (IRID) [1]. However, the prediction accuracy of the SA analyses is still not sufficient and the measured dose rates by the investigations are limited to local areas. Therefore, we developed a method to predict the dose rate distribution in a PCV by combining various numerical calculations with the measured results, and this was applied to the Unit 1 of $1 \mathrm{~F}$.

\section{Estimation of radiation sources in PCV}

There are mainly three kinds of radiation sources in the PCV of 1F. They are 1) radioactive nuclides in fuel debris, 2) inner structural materials activated during normal operations before the accident, 3) all wall surfaces and water in the PCV contaminated with cesium (Cs) released from fuels at the time of accident. These radiation sources were estimated in the following ways.

\subsection{Fuel debris}

Figure 1 shows the calculation scheme to generate the composition of fuel debris. First, a three-dimensional fuel inventory calculation was carried out considering

*Corresponding author. Email: okumura.keisuke@jaea.go.jp axial void and burnup distributions by using the reactor analysis code system MOSRA [2] and its nuclear data library based on JENDL-4.0 [3]. Separately, activation calculations for the structural materials of a fuel assembly were carried out in consideration of impurities using ORLIBJ40 (ORIGEN2 and its revised library with JENDL-4.0) [4].

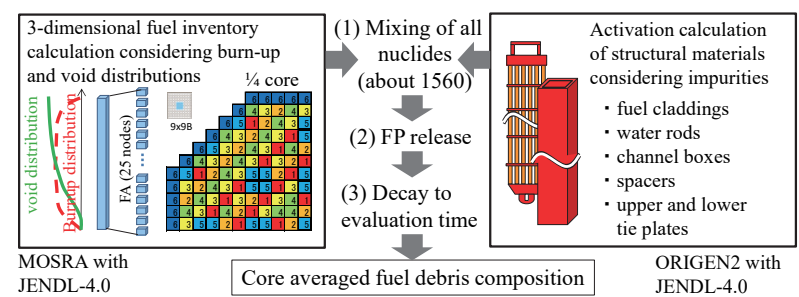

Figure 1. Calculation scheme of fuel debris composition.

After the all nuclides obtained from the above calculations were mixed, fission products (FPs) of noble gases and volatile elements were removed to simulate the release of these gases from the mixture. Two extreme FP release models were employed, as shown in Table 1, with references to the assay result of the TMI-2 fuel debris [5] and a result of FP release behavior tests [6]. Subsequently, for all radionuclides, the decay up to the time of the dose rate estimation was considered. In the present study, this was April 2015 as this is when the dose rate measurement in the PCV of Unit 1 was performed for the first time by a robot (so called "B1 investigation") [7].

The number of photon sources emitted from radionuclides in fuel debris is more than 10,000 in line 
spectra. Since such line spectra are inconvenient for subsequent photon transport calculations, the photon source of fuel debris was represented by a photon source having the 18-energy-group structure of ORIGEN2 [8] which is conventionally used for spent nuclear fuels. The group-wise photon source of fuel debris was calculated based on the JENDL decay data file 2015 [9].

Figure 2 shows the photon spectra of fuel debris at the time of the B1 investigation. The difference of two spectra is mainly due to the release amount of Cs $\left({ }^{134} \mathrm{Cs}\right.$ and $\left.{ }^{137} \mathrm{Cs}\right)$.

Table 1. Two FP release models.

\begin{tabular}{|c|c|c|c|}
\hline Classification & Element & $\begin{array}{c}\text { High release } \\
\text { model (\%) }\end{array}$ & $\begin{array}{c}\text { Low release } \\
\text { model (\%) }\end{array}$ \\
\hline Noble gas & $\mathrm{He}, \mathrm{Ne}, \mathrm{Ar}, \mathrm{Kr}, \mathrm{Xe}, \mathrm{Rn}$ & 99 & 90 \\
\hline $\begin{array}{c}\text { High volatile } \\
\text { FP }\end{array}$ & $\mathrm{Cs}, \mathrm{I}, \mathrm{Te}, \mathrm{Sb}$ & 99 & 60 \\
\hline $\begin{array}{c}\text { Middle volatile } \\
\text { FP }\end{array}$ & $\mathrm{Ru}, \mathrm{Mo}, \mathrm{Sr}, \mathrm{Ba}, \mathrm{Pd}, \mathrm{Rh}$ & 99 & 0 \\
\hline
\end{tabular}

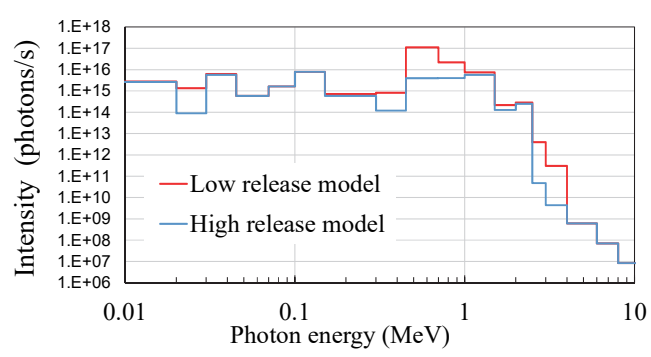

Figure 2. Photon spectra of fuel debris in April 2015.

\subsection{Activated structural materials}

As radiation sources of structural materials by long-term reactor operations, we considered shroud, upper grid plate and lower core support plate, close to the core.

The neutron flux of the shroud was approximated by that at core peripherals calculated by MOSRA with a two-dimensional horizontal core model shown in the left of Figure 1. As for the upper grid plate and lower core support plate, a continuous-energy Monte Carlo calculation code MVP-II [10] was used to estimate neutron fluxes at the top and bottom parts of a fuel assembly with horizontally reflective boundary conditions. Using the obtained neutron fluxes and the reactor operation history [11] of Unit 1 shown in Figure 3, ORIGEN2 calculations were carried out to estimate the amount of activation products in each material. The irradiation period is about 9 years and 4 months from November 2001 when the reactor was restarted after shroud exchange, until March 2011 when the accident occurred. The capacity factor of Unit 1 in this period is low as about $48 \%$.

As a result of activation calculations, it was found that ${ }^{60} \mathrm{Co}$ was main contributor to the dose rate at the time of the B1 investigation by $\gamma$-radiation, and others (e.g. ${ }^{55} \mathrm{Fe},{ }^{63} \mathrm{Ni},{ }^{54} \mathrm{Mn}$ ) were negligible compared with
${ }^{60} \mathrm{Co}$. Most of ${ }^{60} \mathrm{Co}$ is generated from $(\mathrm{n}, \gamma)$ reactions of ${ }^{59} \mathrm{Co}$ existing in structural materials as an impurity. Therefore it can be said that the uncertainty of the activation source is proportional to the initial concentration of Co. In this study, the concentration of Co was set to $305 \mathrm{ppm}$.
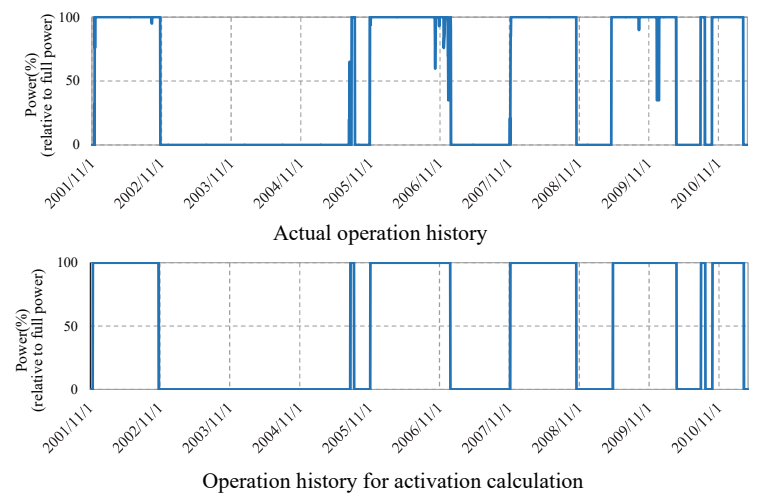

Figure 3. Actual reactor operation history (upper) and simplified model for activation calculation (lower).

\subsection{Cs contamination}

The $\mathrm{Cs}$ concentration and $\mathrm{Cs}$ isotopic ratio $\left({ }^{134} \mathrm{Cs} /{ }^{137} \mathrm{Cs}\right)$ in water inside the PCV were determined based on the result of a sample analysis from the residence water of Unit 1 in October $2012 .{ }^{134} \mathrm{Cs}$ and ${ }^{137} \mathrm{Cs}$ were disintegrated until the time of the B1 investigation. Since the effect of decontamination of circulating water is not considered, the concentration may have been set higher than the actual.

Cs contamination other than water, for example, the amounts of Cs on the wall surfaces of drywell, pedestal, reactor pressure vessel (RPV), etc. were roughly estimated based on the result of the SA analysis by IRID [1]. In the SA analysis, the short-term transition (within 6 days) of FP elements is calculated. Therefore, assuming that all Cs estimated by the SA analysis does not move after that, only the disintegration effect of radioactive $\mathrm{Cs}$ until the time of the $\mathrm{B} 1$ investigation was considered. In addition, it was assumed that the isotopic ratios ${ }^{134} \mathrm{Cs} /{ }^{137} \mathrm{Cs}$ of all $\mathrm{Cs}$ contaminants are the same as that in the residence water.

The Cs contamination based on the SA analysis is not related with the previously mentioned two FP release models to estimate the radiation sources of fuel debris.

\section{Prediction of dose rate distribution}

\subsection{Calculation model and method}

A series of dose rate calculations in this study was carried out using a Monte Carlo calculation code PHITS [12]. Figure 4 shows the three-dimensional model of Unit 1 for the PHITS calculation in this study. In this calculation model, the three types of radiation source described in the previous chapter are set in the PCV. The distribution and amounts of fuel debris are based on the 
result of the SA analysis by IRID [1]. However, the erosion of concrete by fuel debris at the bottom of PCV was ignored for simplicity.

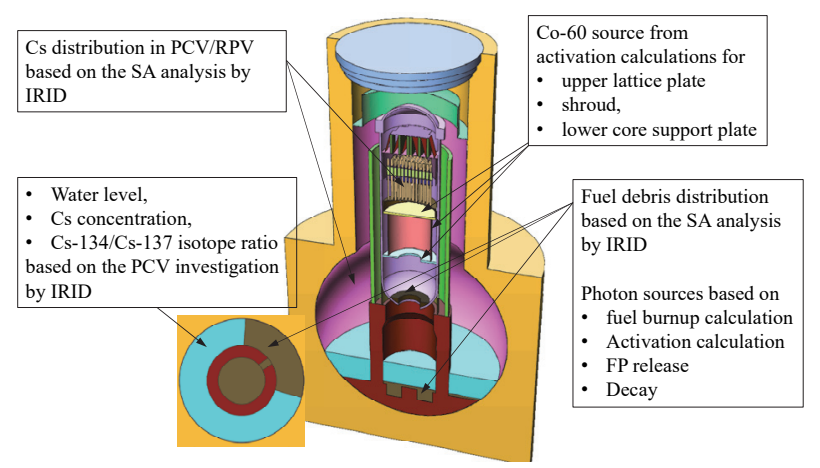

Figure 4. Dose rate calculation model by PHITS (Unit 1).

The uncertainty of the radiation sources is still large, and it is thought that it will be changed by future improvement of the SA analysis and progress of the PCV investigations. Therefore, all the radiation sources in PCV were divided into $N$ pieces, and the response to the dose rate distribution $d_{i}(\mathbf{r})$ from each unit source $i$ was calculated by PHITS. Using the intensity $S_{i}$ of the $i$-th source, the dose rate distribution $D(\mathbf{r})$ in the PCV is expressed by the following equations.

$$
\begin{aligned}
& D(\mathbf{r})=\sum_{i}^{N} S_{i} d_{i}(\mathbf{r}), \\
& d_{i}(\mathbf{r})=\int H^{* 10}(E) \Phi_{i}(\mathbf{r}, E) d E,
\end{aligned}
$$

where, $H^{* 10}(E)$ is the flux-to-dose equivalent rate conversion coefficient based on ICRP-74 [13], and $\Phi_{i}(E)$ is a photon spectrum calculated by PHITS for the $i$-th source.

Although the number of radiation sources $(N)$ can be arbitrarily determined from the number of sensitivities of the radiation sources we want to know, it is limited from the amount of information available from the results of SA analysis and PCV investigations. In this study, we set as $N=63$, they are two fuel debris distributed inside and outside RPV, three activated structural materials in the RPV (shroud, upper grid plate and lower core support plate) and 58 of Cs contaminations, in which ${ }^{134} \mathrm{Cs}$ and ${ }^{137} \mathrm{Cs}$ with different half-lives are separately considered because it is convenient to predict the time change of $D(\mathbf{r})$.

We assumed that the walls and floor under the water were contaminated with the Cs with the same concentration to that on the lower drywell in the air, although the actual situation is unknown.

In the Monte Carlo calculation by PHITS, 200 million photons were traced to get $d_{i}(\mathbf{r})$ for each radiation source.

\subsection{Results and discussions}

Figure 5 shows the typical results of the contributions $\left(S_{i} d_{i}(\mathbf{r})\right)$ to the dose rate distribution in
PCV. The predicted dose rate obtained by Eq.(1) is shown in Figure 6.
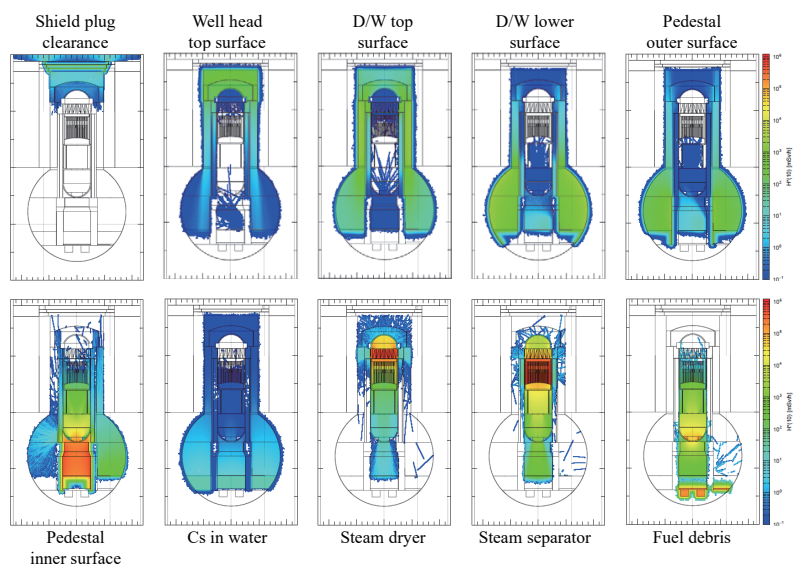

Figure 5. Source-wise dose rate distribution.

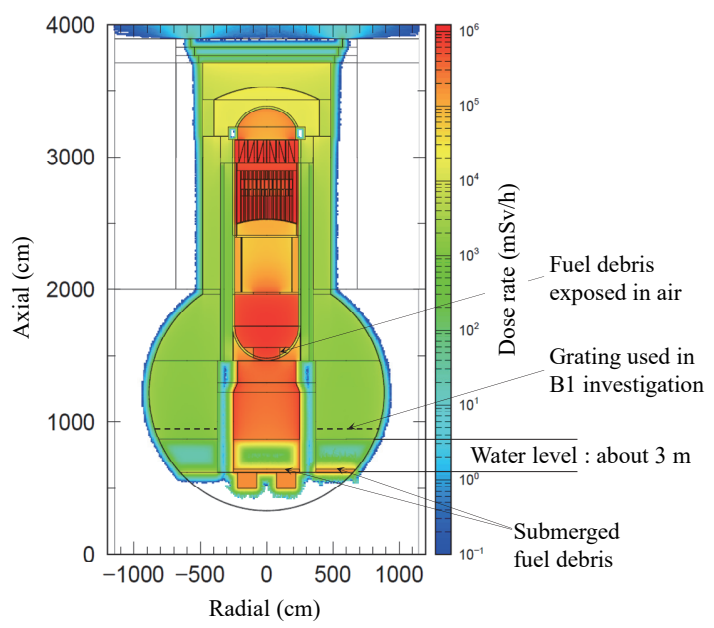

Figure 6. Predicted dose rate distribution of Unit-1 in Apr. 2015 (without adjustment to the measured dose rate in the B1 investigation).

In the $\mathrm{B} 1$ investigation, the dose rates were measured by a robot at 11 points on the grating shown in Figure 6 . The measured dose rates range from 4.7 to $9.7 \mathrm{~Sv} / \mathrm{h}$, with an average of $7.2 \mathrm{~Sv} / \mathrm{h}(1 \sigma=1.4)$. On the other hand, the dose rate near the grating in Figure 6 is about 1.4 $\mathrm{Sv} / \mathrm{h}$.

The difference between the measured and calculated dose rates is considered to be mainly due to the amounts of Cs contaminations by the SA analysis. As shown in Figure 5, the dose rate near the grating position is sensitive to the Cs contaminations on the lower drywell $(\mathrm{D} / \mathrm{W})$ and on the outer surface of pedestal, and not so sensitive to other radiation sources. Therefore, the amounts of Cs outside the RPV were adjusted so that the dose rate at the grating position matched the measured average value. In this procedure, the values of $S_{i}$ in Eq.(1) corresponding to Cs contaminations on the all wall surfaces outside the RPV were multiplied by the same adjustment factor $(=7.2 / 1.4)$. The modified dose 
rate distribution is shown in Figure 7.

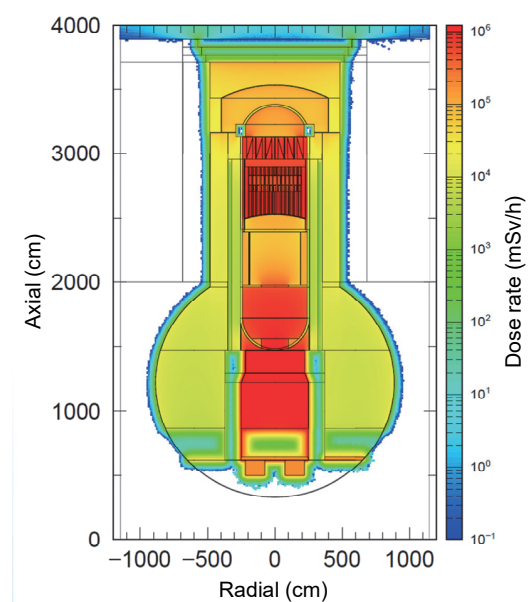

Figure 7. Predicted dose rate distribution of Unit-1 in Apr. 2015 (with adjustment to the measured dose rate in the B1 investigation).

From these results, we can say the followings.

(1) There is a possibility that the dose rate in the RPV is quite high especially near the steam dryer, water-steam separator and bottom of the RPV due to their Cs contaminations. However the uncertainty of the dose rate in the RPV is large because of no available measurements. The large dose rate will make it difficult to retrieve fuel debris by the upper access method when the drywell can not be fully flooded.

(2) The dose rate inside the pedestal in air may be high mainly due to the surface contamination with Cs, but the effect to the outside is limited because of the shielding of pedestal wall.

(3) The dose rate close to the submerged fuel debris is estimated within a few hundred $\mathrm{Sv} / \mathrm{h}$ in consideration with the self-shielding of $\gamma$-radiation in the fuel debris and the volatile FP release (low FP release model is used in Figure 6).

(4) It is difficult to confirm the distribution of fuel debris from the measurement results of the B1 investigation. This is because, as shown in Figure 5, the dose rate near the grating is not sensitive to the $\gamma$-radiation from the fuel debris.

(5) The dose rate in water is higher near the water surface, lower in the center, higher again near the bottom of the PCV. Whether there are fuel debris or not at the bottom of the PCV, this tendency will be observed if the bottom is contaminated with Cs. Therefore, to confirm the existence of fuel debris deeply submerged in water, it is effective to detect $\gamma$-rays unique to fuel debris by using a sensor which has an energy resolution capability. For example, target nuclides are ${ }^{154} \mathrm{Eu}$, ${ }^{144} \mathrm{Ce}+{ }^{144} \mathrm{Pr}$, and ${ }^{60} \mathrm{Co}$, which are expected to coexist with actinides and emit $\gamma$-rays whose energies are higher $(>1 \mathrm{MeV})$ than those of ${ }^{134} \mathrm{Cs}$ and ${ }^{137} \mathrm{Cs}$. Another way is to detect spontaneous neutrons mainly emitted from ${ }^{244} \mathrm{Cm}$. These approaches are under study collaborated with Lancaster and Manchester universities [14].
As shown the above, the present method is effective not only for forming an adequate decommissioning strategy of $1 \mathrm{~F}$, but also for obtaining information necessary for future PCV investigations.

\section{Conclusion}

We propose a method to predict the most probable dose rate distribution in the PCV of $1 \mathrm{~F}$. In this method we utilize the available results of the PCV investigations and those of numerical calculations such as: severe accident analyses, irradiation calculations of fuel/ structural materials and photon transport Monte Carlo calculations. From a result of applying it to Unit 1 of $1 \mathrm{~F}$, the method is considered useful to determine $1 \mathrm{~F}$ decommissioning strategy and future planning of PCV investigations.

\section{Acknowledgements}

This work was funded by the Ministry of Education, Culture, Sports, Science and Technology (MEXT) and the Engineering and Physical Sciences Research Council (EPSRC: EP/N017749/1) in the U.K. Malcolm J. Joyce acknowledges the support of a Royal Society Wolfson Research Merit award.

\section{References}

[1] IRID and IAE, FY2016 Report of Subside Program "Project of Decommissioning and Contaminated Water Management (Upgrading Level of Grasping State inside Reactor)", (2017), available on IRID web site: http://irid.or.jp/en/reports/ [in Japanese]

[2] K. Okumura, K. Kojima, T. Okamoto, et al., Nuclear data for severe accident analysis and decommissioning of nuclear power plant, Proc. 2012 Symposium on Nuclear Data, Kumatori, Japan, Nov. 15-16, 2012, JAEA-Conf 2013-002 (2013), pp.15-20.

[3] K. Shibata, O. Iwamoto, T. Nakagawa, et al., JENDL-4.0: A new library for nuclear science and engineering, J. Nucl. Sci. Technol. 48 (2011), pp.1-30.

[4] K. Okumura, K. Sugino, K. Kojima, et al., A set of ORIGEN2 cross section libraries based on JENDL-4.0: ORLIBJ40, JAEA-Data/Code 2012032, (2013). [in Japanese].

[5] H. Uetsuka, F. Nagase, T. Suzuki, Gamma spectrometry of TMI-2 debris, JAERI-Research 95-084, (1995). [in Japanese]

[6] P.D.W. Bottomley, A-C. Gregorire, P. Carbol, et al., Fission product and actinide release from the debris bed test PHEBUS FPT4: Synthesis of the post test analyses and of the revaporisation testing of the plenum samples, Nucl. Eng. and Technol. 38 (2006), pp.163-174.

[7] Tokyo Electric Power Company, Development of a technology to investigate inside the reactor primary containment vessel (PCV) - Results of site test 
"Investigation B1" on grating around the pedestal inside Unit 1 PCV -, available on the website of TEPCO, April 30, (2015).

[8] A.G. Croff, ORIGEN2: A versatile computer code for calculating the nuclide compositions and characteristics of nuclear materials, Nucl. Technol. 62 (1983), pp.335-352.

[9] J. Katakura and F. Minato, JENDL Decay Data File 2015, JAEA-Data/Code 2015-030 (2016).

[10]Y. Nagaya, K. Okumura, T. Mori, M. Nakagawa, MVP/GMVP II: General purpose Monte Carlo codes for neutron and photon transport calculation, JAERI 1348 (2005).

[11]JNES, Nuclear Facility Operation Management Annual Report, (2000 2012). [in Japanese]
[12]T. Sato, K. Niita, N. Matsuda, et al., Particle and heavy ion transport code system PHITS, Version 2.52, J. Nucl. Sci. Technol. 50 (2013), pp.913-923.

[13]ICRP, Conversion coefficients for use in radiological protection against external radiation, ICRP Publication 74, Ann. ICRP 26 (3-4), (1996).

[14]M. Nancekievill, A.R. Jones, M.J. Joyce, B. Lennox, S. Watson, et al., A remote-operated system to map radiation dose in the Fukushima Daiichi primary containment vessel, Proc. Int. Conf. on Advancements in Nuclear Instrumentation Measurement Methods and their Applications (ANIMMA2017), June 19-23, 2017, Liege, Belgium (2017). ANIMMA-2095 [electric file] 